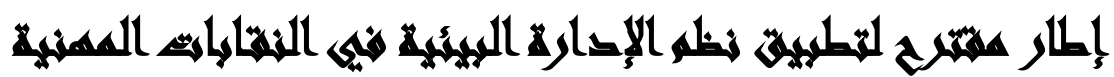

[17]

\author{
خالد حسين أحمد(')- محمد سالم محمود(')- عصام عبد الرازق عبده(r)

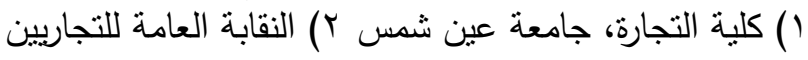

\section{المستخليك}

يهذف الباحثون من خلال هذه الدارسة إلى وضع إطار مقترح لتطبيق نظم الإدارة البيئية

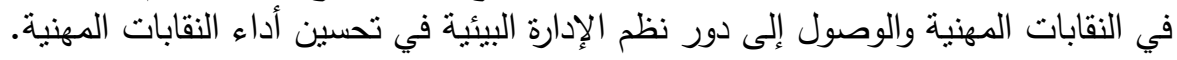

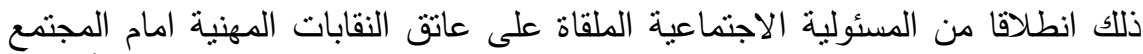

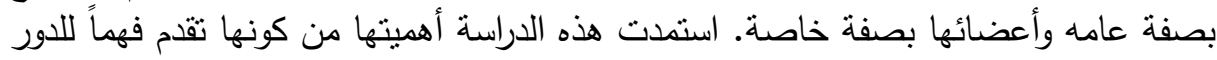

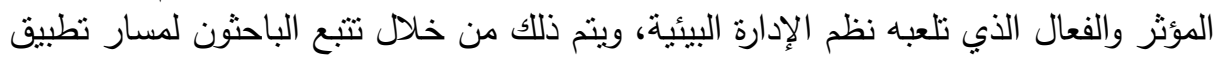

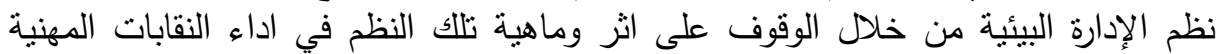
ومحاولة الوصول إلى مظاهر ذللك الأثر على النقابات المهنية ودورها المجتمعي المنوطة به الهاه

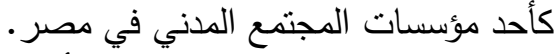

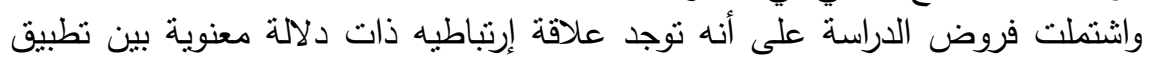

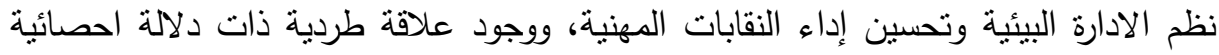

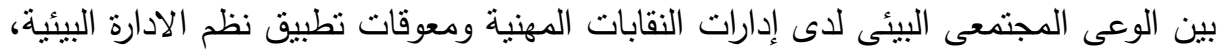

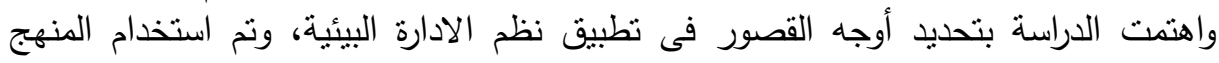

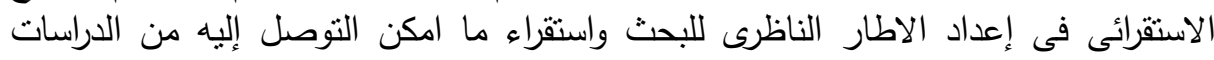

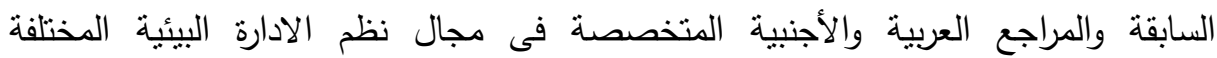

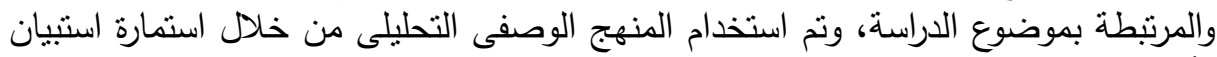

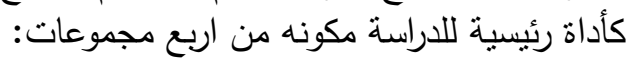

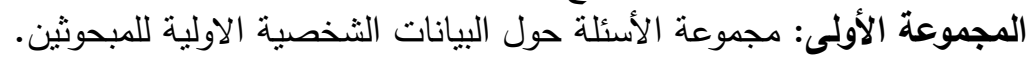
المجموعة الثانية: مجموعة الأسئلة المستخدمة لقياس نطبيق نظم الإدارة البيئية فى النقابات

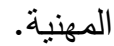
المجموعة الثالثة: مجموعة الأسئلة المستخدمة لقياس دور نظم الإدارة البيئية فى تحسن اداء

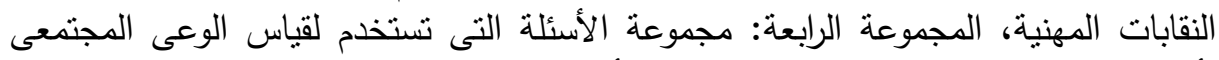

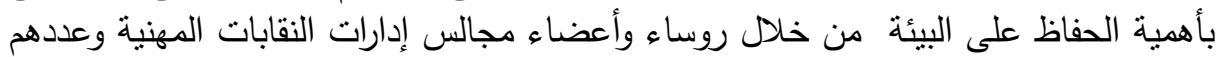

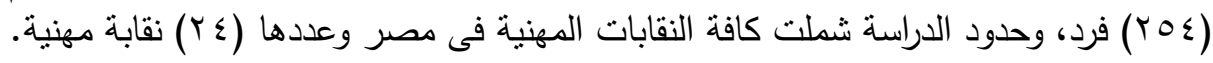

$$
\text { المجلد السادس والثلاثون، الجزء الأول، ديسمبر } 17 \text { ـ }
$$


توصلت الدراسة الى صحة الفروض وهي وجود علاقة إرتباطيه ذات دلالة معنوية بين

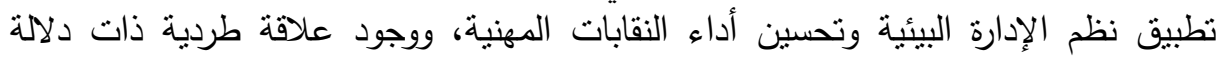

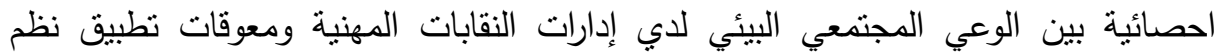
الإدارة البيئية، ونم التوصية بضرورة تضمين الإدارة البيئية ضمن خطة عمل النقابات البات الئات المهنية،

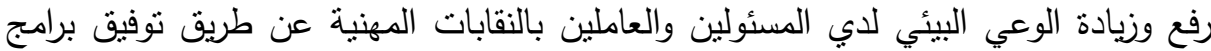
بيئية تثقيفية بأخطار أضرار تلوث البيئة، وأهمية وكيفية الحفاظ على لبئ البيئة.

\section{Xas}

تلعب النقابات المهنية دوراً فعالاً كأحدي اهم واكبر مؤسسات المجتمع المدني في مصر ، لما يمكن أن تقوم به في تفعيل مشاركة عدد اكبر من المواطنين في تقرير مصائرهم والتفاعل

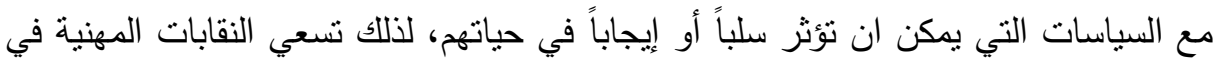
مصر إلى خلق دور مؤثر وفعال في المجتمع يهدف إلى التنمية مع وجود علاقة منوازنة بينها وبين الحكومة أساسها الاحترام المتبادل.

النقابات المهنية في مصر شأنها شأن اي مؤسسة اخري تؤثر وتتأثر بعوامل البيئة المحيطة بها، وتتأثر القرارات الإدارية لأي مؤسسة بعدة عوامل بيئية من شأنها ان تحقق لتقان

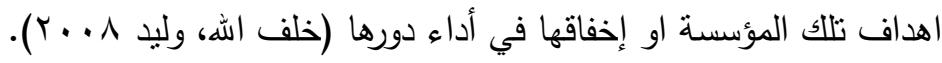
قد تعددت المشاكل البيئية وتنوعت وأصبحت تشكل خطراً حقيقا على الإنسان وعلي الحياة برمتها، وذلك بفعل الأنشطة البشرية المختلفة التي لم نراعي الاعتبارات البيئية وركزت البتان

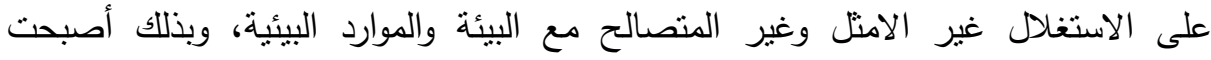
المشكلات البيئية واحده من أهم المشكلات الكونية المعاصرة التي اهتم بها الفكر الاقتصادي،

$$
\text { الإداري، الاجتماعي، السياسي ... الخ. }
$$

عليه تولت قضايا البيئة والإدارة البيئية اهتمام متزايد منذ مطلع القرن التاسع عشر ، حيث بذلت العديد من الجهود الوطنية والإقليمية والدولية التي ركزت على قضايا البيئة والإدارة 
لما كان المنظمات الخدمية واحدة من المكونات الرئيسية للتنمية والتى تؤثز انشطتها المختلفة على البيئة بوجه عام من خلال الاثار الناتجة عن انشطنها التي تضر بالبيئة بمختلف مكوناتها.

قد برز الاهنمام بوجود انظمه بيئية تهنم بإدارة البيئة وتوجيه وضبط أنشطة هذه

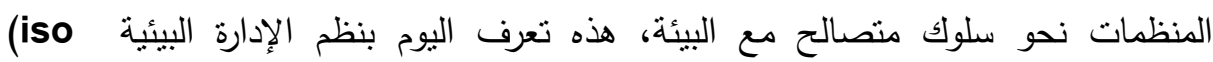

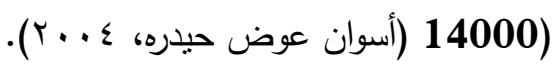

من هذا المنطلق تم اختيار موضوع الدراسة للوقوف اولا على مختلف المفاهيم والرؤى النظرية المتصلة بالبيئة والإدارة البيئية، من ثم الوقوف على واقع الإدارة البيئية في النقابات المهنية في محاولة لصياغة نظام مقترح يمكن النقابات المهنية في مصر من تطبيق نظم

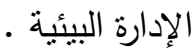

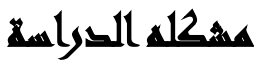

قام الباحثون بدراسة استطلاعية للوقوف على الوضع البيئى داخل النقابات المهنية، وقد قامت الدراسة على سؤال رئيسى: هل تطبق النقابات المهنية فى مصر نظم الادارة البيئية وذللك من خلال استمارة استبيان وكانت نتائج الدراسة كالتالى: جدول رقم(1): توزيع عينة الدراسة لإستجابات عينة الدراسة على التساؤل هل نطبق النقابات

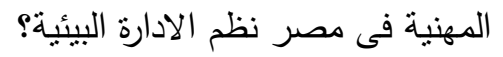

\begin{tabular}{|c|c|c|}
\hline النسبة & العدد & الإجابة \\
\hline$\wedge 9, \wedge$ & TYA & $\gamma$ \\
\hline$\Lambda, V$ & YY & لا أعلد \\
\hline 1,7 & $\varepsilon$ & نعر \\
\hline $1 \ldots$ & YOS & الإجمالى \\
\hline
\end{tabular}

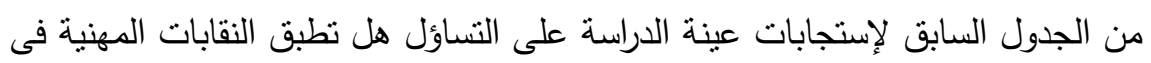

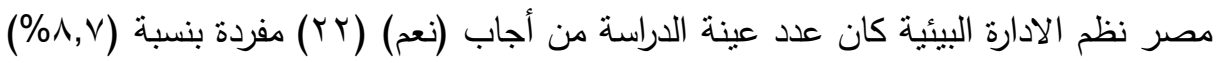

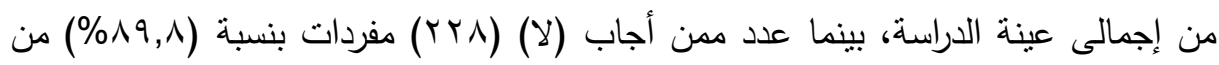


اجمالى عينة الدراسة هى النسبة الأعلى، بينما من أجاب (لا أعلم) (ع مفردة بنسبة (T, 1\%) من إجمالى عينة لدراسة، وهنا وضحت مشكلة الداسهة الدراسة. تتبلور مشكلة الدراسة في وضع اطار مقترح لنطبيق نظم الإدارة البيئية فى النقابات

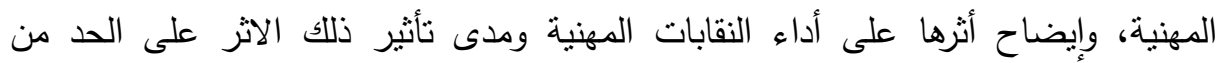
معوقات اداء النقابات المهنية، لما لها من دور نقافي واجتماعي وسياسي وخدمي.

\section{أسهولغ الصواسم}

1- ما مدى امتلاك إدارات النقابات المهنية وعى مجتمعى بأهية الحفاظ على البيئة ؟ ז- ما مدى اهتمام إدارات النقابات المهنية بتضمين مكون الإدارة البيئية ضمن استراتيجية

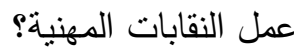
ب- ما معوقات نطبيق نظم الإدارة البيئية فى النقابات المهنية؟

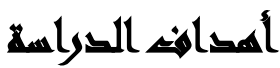

يحاول الباحثون من هذه الاراسة تحقيق الاهداف الاتية: أ- الهرف الرئيسى: وضع إطار مقترح لتطبيق نظم الإدارة البيئية في النقابات المهنية. ب - الاهداف الفرعية 1- تحسين مستوى الاداء البيئى فى النقابات المهنية. r- التعرف على معوقات تطبيق نظم الإدارة البيئية في النقابات المهنية.

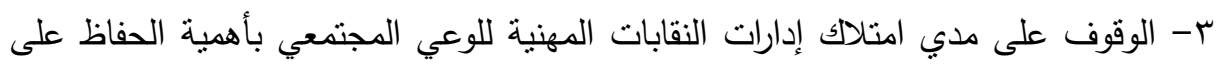

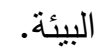

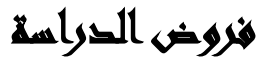

$$
\text { يمكن التعبير عن فروض الاراسة كما يلي: }
$$

ا- توجد علاقة ذو دلالة إحصائية بين تطبيق نظم الإدارة البيئية وتحسين أداء النقابات 
خالد حسين أحمد وآخرون

r- توجد علاقة ذو دلالة إحصائية بين الوعي المجتمعي البيئي لدي ادارات النقابات المهنية ومعوقات تطبيق نظم الإدارة البيئية.

\title{
كتغيرايت التراسلة
}

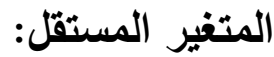 \\ - وضع إطار لتطبيق نظم الادارة \\ - تحسين الاداء \\ المتغير التابع: لادارة البيئية
}

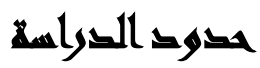

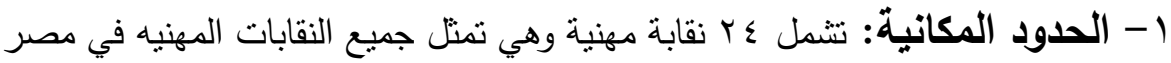

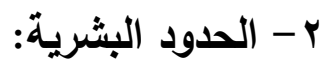
- رؤساء النقابات المهنية - أعضاء مجالس النقابات المهنية

r- الحدود الزمنية: شاملة الدراسة تحليل واقع بيانات نطبيق نظم الإدارة البيئية في

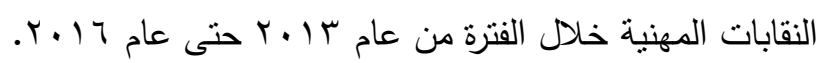

\section{أهمية الصراسة}

تتمثل أهمية هذه الدراسة من الناحيتان العلمية والعملية:

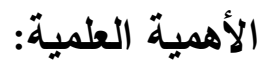
- وضع إطار لتطبيق نظم الادارة البيئية فى النقابات المهنية . - معوقات تطبيق نظم الادارة البيئية

الأهمية العملية: التوقع ان تساهم هذه الدراسة فى إلقاء الضوء على السلبيات الضارة بالبيئة والمؤثزة على أداء النقابات المهنية، وعرض بعض التوصيات للحد من هذه السلبيات

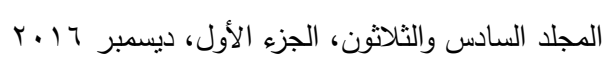




\section{مخاهيه التراسة}

أ- الإدارة البيئية:

1-الإدارة البيئية هي أسلوب تفكير مختلف وغير تقليدي في إدارة تشغيل المنشات المختلفة

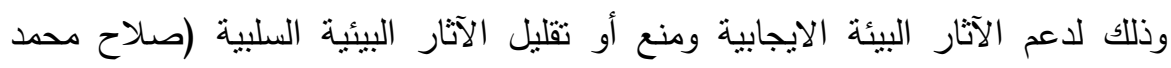

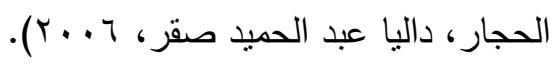
r-عرف كونتز الإدارة على إنها وظيفة تتفيذ الأعمال عن طريق الآخرين (محمد سالم،

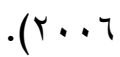
ب-الإدارة البيئية امتداد لهفهوم الإدارة بمعناه العام وخاصة عند تطبيقه في مجالات معينة

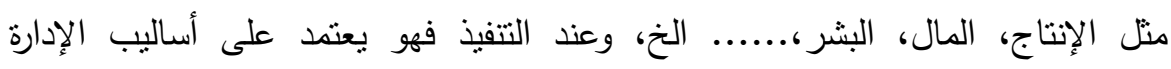

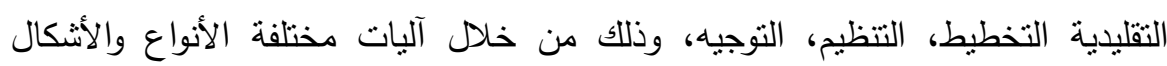
لتحقيق أهدف محددة وتقييم الأداء، ثم تصحيح المسار (مقدم وهيبه، ( . . ب). ب- نظام الإدارة البيئية:

1- يعرف نظام الإدارة البيئية بأنه هيكل لإدارة المنظمات من أجل تقييم تأثنيراتها البيئية كونها جزءا من نظام الإدارة في المنظمة يستخدم في تطوير وتتفيذ السياسة البيئية. (الأكاديمية

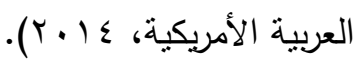

r- نظام إدارة البيئة (EMS) هو نظام يشير إلى إدارة البرامج البيئية بالمنشاة شاملا الهيكل

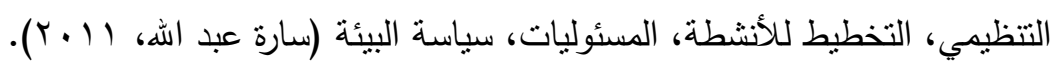

$$
\text { ج- النقابات المهنية: }
$$

ا- النقابة باللغة الاتجليزية Syndicate تعنى الرئاسة ويقال لكبير القوم نقيباً أو رئيساً أو عقيداً. r- هي جمعية نتكل لأغراض المفاوضة الجماعية أو المساومة الجماعية بشأن شروط

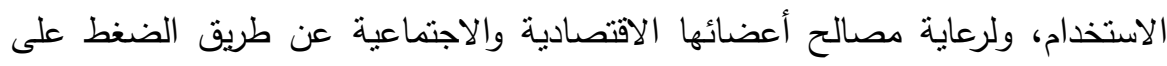


الحكومات والهيئات التتريعية والالتجاء إلى العمل السياسي فى حالات معينة (وليد سيد

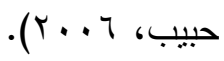

r- النقابة هي عبارة عن إتحاد للمشتغلين فى مهنه أو صناعة أو حرفة مرتبطة بعضها ببعض بهدف حماية مصالح المهنيين والعمال والحرفيين والدفاع عن حقوقهم وتحسين

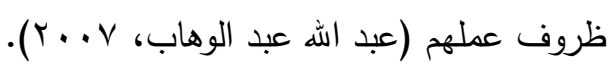

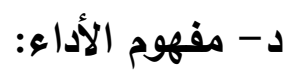

1 - هو المخرجات والأهداف التي تسعى المنظمة إلى تحقيقها عن طريق العاملين فيها. r- هو العملية التي يتعرف من خلالها على أداء الفرد لههامه وقدراته على الأداء والخصائص اللازمة لنأدية العمل بنجاح. r- المسئوليات والواجبات والأنشطة والمهام التي يتكون منها عمل الفرد الذي يجب لبه عليه

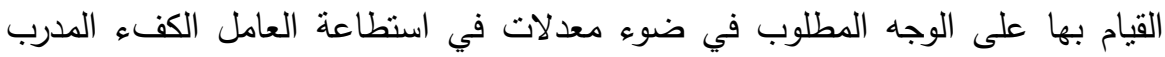
القيام بها (Carter tony, 2009). ع - عرف Andrewd الأداء بأنه تفاعل لسلوك الموظف وأن ذلك السلوك يتحدد بتفاعل جهد

$$
\text { وقدرات الموظف فى منظمته. }
$$

ه- عرف هاينز Haynes الأداء بأنه الناتج الذي يحققه الموظف عند قيامه بأى عمل من

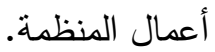

צ- عرف Fred الأداء بحسب نتائج السلوك الذي رافق ذلك الأداء، فيرى أن الأداء هو نتيجة

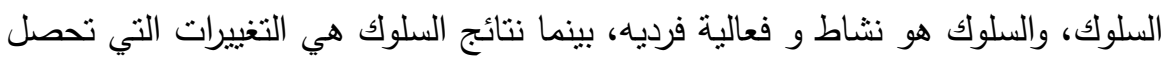

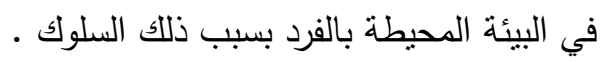

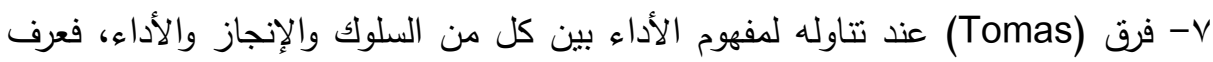

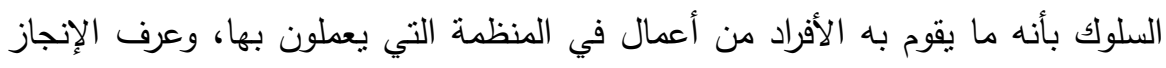

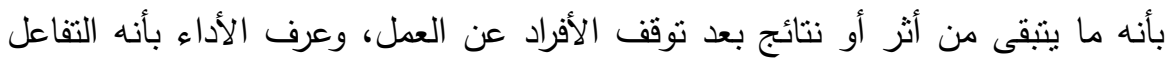

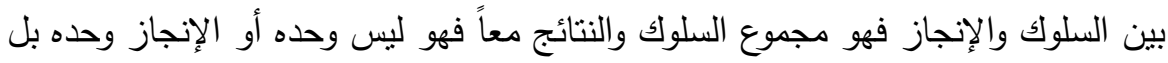
هو تكاملهما معاً (Apter, David, 2006)

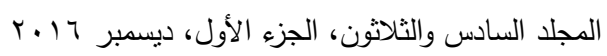




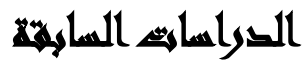

ا .دراسة شوقي محمد سليمان عطية(1 1 • r): تهدف هذه الدراسة إلى التعرف على نظم الإدارة البيئية في ظل العلاقات النتظيمية لأجهزة الإدارة المحلية، وتحديد مدي قيام أجهزة الإدارة المحلية بتطبيق هذه النظم، بجانب تقييم فاعلية تطبيق نظم الإدارة البيئية في

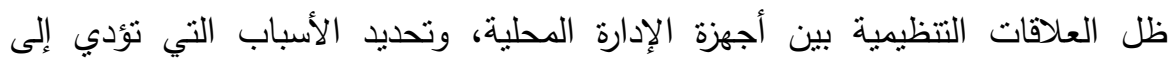
القصور في نظم الإدارة البيئية وتطبيقها في ظل العلاقات التظظيمية بين أجهزة الإدارة المحلية وكيفية معالجنها. توصلت الاراسة إلى النتائج والآتية: ا ـ لا يوجد فكر بيئي أو المام كامل لاي الادارة العليا المحلية والعاملين بها عن مفهوم الإدارة البيئية وأهدافها وسياستها ومنطلبات تحقيقها. r. ضعف الإحساس لدي الإدارة العليا بالحاجة لتطبيق نظم الإدارة البيئية. r. ضعف التتسيق والتعاون بين ادارات البيئة ومديريات الخدمات بالمحافظات.

يري الباحث: أنه يمكن لدراستتا الحالية الاستفادة من نتائج تللك الدراسة حول عدم وجود فكر عن مفهوم الإدارة البيئية بجانب ضعف التتسيق والتعاون بين ادارات البيئة ومديريات الخدمات

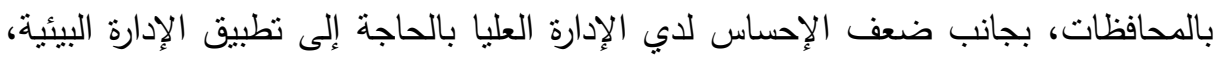
وهو الأمر الذي تهنم به دراستتا الحالية. r - دراسة محمد عبد المنعم إبراهيم(1 . . r): تهدف هذه الدراسة إلى التعرف على

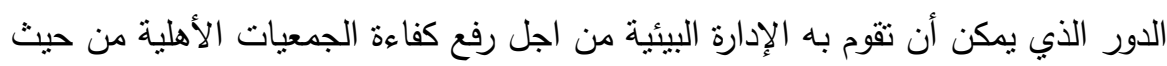

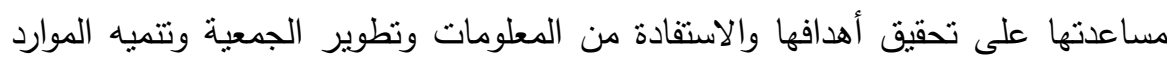
البشرية وتوفير الموارد المادية. توصلت الدراسة إلى النتائج الآتية: ا ـ أهمية تطبيق الإدارة البيئية داخل الجمعيات الأهلية. r. إن تطبيق الإدارة البيئية يساهم إلى حد كبير في مساعدة الجمعيات على تحقيق أهدافها. 
يرى الباحثون أنه يمكن لدراستتا الحالية الاستفادة مما توصلت اليه هذه الدراسة من نتائج

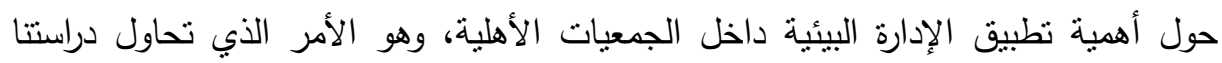
الحالية تطبيقه داخل النقابات المهنية والتي تعتبر من كبري مؤسسات المجتمع المدني.

\section{الإيار المنهيبيه للسوراسلا}

- منهج الاراسة: تعنبر خطوه اختيار المنهج الملائم للاراسة من أهم الخطوات التى تساعد على انجاز البحث على النحو الذى يحقق أهدافه لأن المنهج يساعد الباحثون على الى اهن تحقيق أهدافه التى يسعى للوصول إليها و بالتالى الاجابة على تساؤلات بحثه ومن خلاله

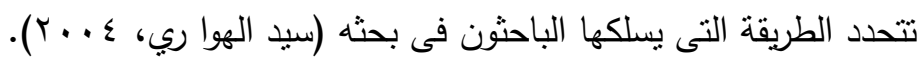

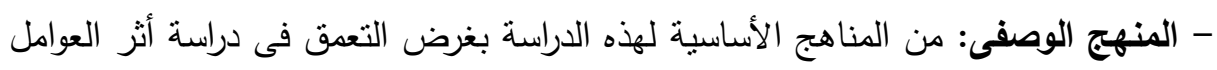
البيئة على أداء النقابات المهنية. كما أن من شأن هذا المنهج الكثف عن علاقة العوامل البيئية وفثل أو نجاح النقابات المهنية فى أداء دورها وتقييم أداء النقابات لدورها المجتمعي والسياسي والثقافي والذى من شأنه

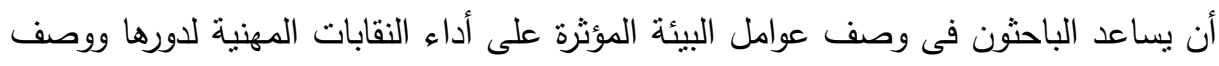
الظروف والعوامل المؤثرة فى أداء النقابات لدورها المنوطة بهاء

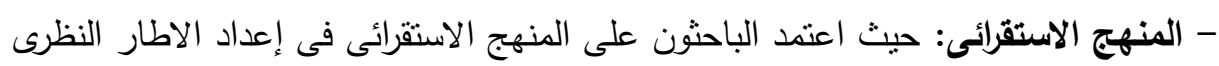

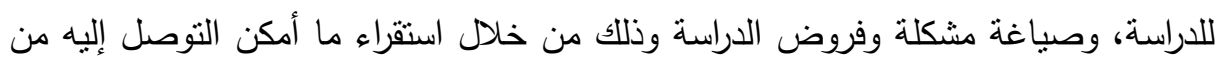

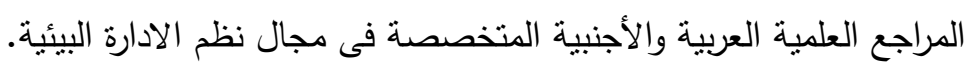

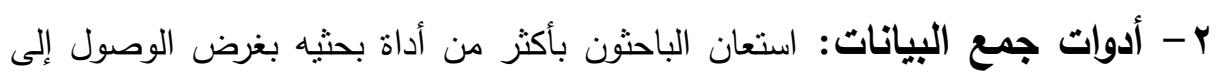
بيانات دقيقة ومنعمقة ومن أهم الأدوات المستخدمة أداة.

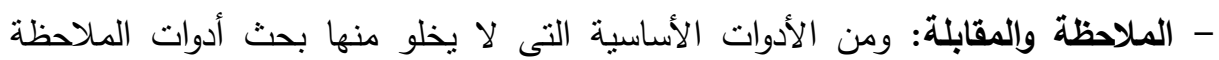

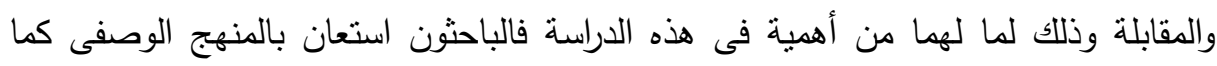
اشار من قبل بغرض وصف عوامل البيئة ومدى تأثيرها على أداء النقابات المهنية لدورها، 
وبالتالي سوف تقدم أداه الملاحظة مساعدة كبيرة فى هذا الوصف كما نساعد أداة المقابلة الباحثون فى الحصول على المعلومات.

\section{هيتمع التواسمة}

تكون مجتمع الدراسة من رؤساء وأعضاء مجالس إدارات النقابات المهنية والبالغ عددهم

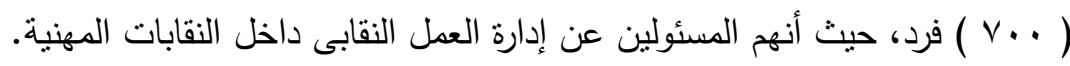

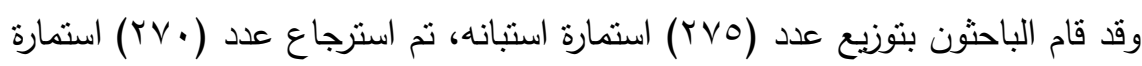

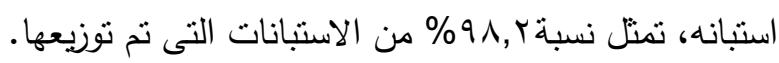

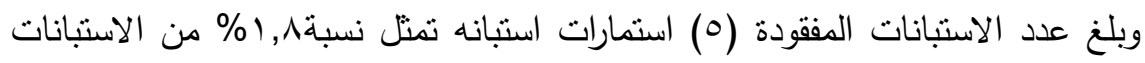

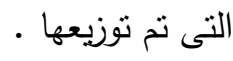

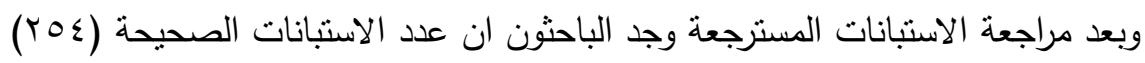

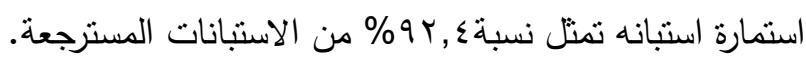

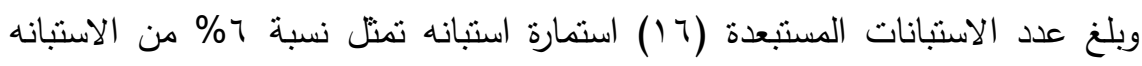
المسترجعة.

\section{أسلوهب الىراسها}

استخلم الباحثون منهجا متكاملا من الجانب النظري والجانب التطبيقي أ. الجانب النظري: اعتمد الباحثون في جمع بيانات الجانب النظري على الكتب والدوريات العربية والأجنبية والرسائل الجامعية والبحوث التي تم الحصول عليها عن طريق الانترنت

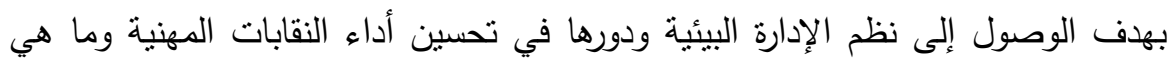
معوقات تطبيقها.

ب. الجانب التطبيقي: ذلك بجمع البيانات من خلال الدراسة الميدانية المتمنكة في استمارة اسنتيان، باختيار عينة من قيادات النقابات المهنية المسئولين عن ادارة العمل النقابي في جميع النقابات المهنية وذلك بهدف: باندان 


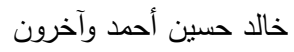

- دراسة العلاقة بين تطبيق نظم الادارة البيئية وتحسين أداء النقابات المهنية. - تحديد الإطار الملائم لنطبيق نظم الادارة البيئية في النقابات المهنية.

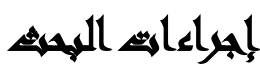

أداة البحث: نم تطبيق استمارة استبيان مكونة من أربع مجموعات: المجموعة الأولي: مجموعة الأسئلة حول البيانات الثخصية الاولية للمبحوثين: وتحتوي على أسئلة تستخدم لقياس كل من: (السن، النوع، اسم النقابة، الموقع النقابي، سنوات الخبرة النقابية، المستوي التعليمي، الحالة الاجتماعية). المجموعة الثانية: مجموعة الأسئلة المستخدمة لقياس تطبيق نظم الإدارة البيئية في النقابات المهنية: تحتوي على أسئلة نستخدم لقياس كل من: (تطبيق النقابات المهنية لنظم الإدارة البيئية، مجالات النطبيق، الفائدة التي تعود على النقابات نتيجة لنطبيق نظم الإدارة البيئية، أسباب عدم التطبيق، معوقات النطبيق، سياسة النطبيق، الدعم اللازم للتطبيق). المجموعة الثالثة: مجموعة الأسئلة المستخدمة لقياس دور نظم الإدارة البيئية في تحسين اداء النقابات المهنية: تحتوي على أسئلة تستخدم لقياس كل من (تأثير تطبيق نظم الإدارة البيئية على تحسين البيئة الداخلية للنقابات المهنية، امتلاك النقابات المهنية للمقومات المادية والبشرية لتطبيق نظم الإدارة البيئية).

المجموعة الرابعة: مجموعة الأسئلة التي تستخدم لقياس الوعي المجتمعي بأهمية الحفاظ

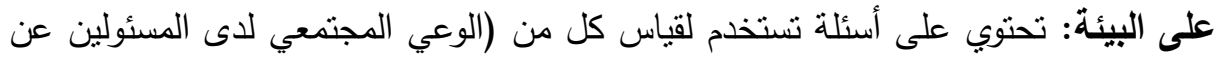

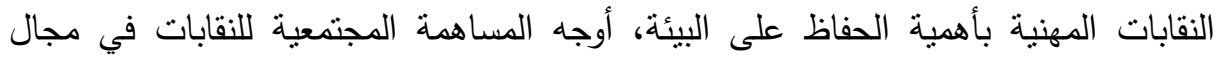

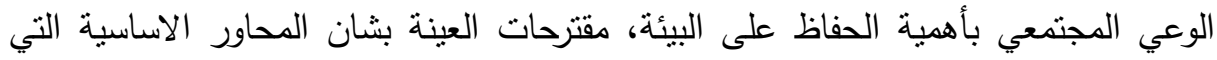
يجب ان يتضمنها الإطار المقترح لنظام الإدارة البيئية الملائم لطبيعة النقابات المهنية). قياس الاعتمادية (ثبات وصدق استمارة الاستبيان): 
أ. ثبات الاستيبان: للتحقق من ثبات الاستبيان استخدم الباحثون معامل الفا كرونباخ والتي اوضحت أن قيم الثبات جميعها قيم مرتفعة حيث بلغت قيم (Alpha Cronbach)

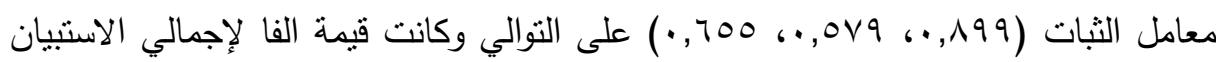
(PVV)

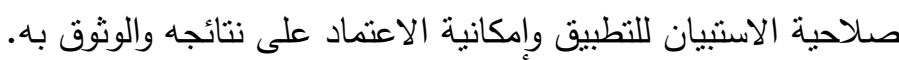
ب. صدق الاتساق الاخلي للاستيّان : من صدق الاتساق للاسنبيان نجد ان معامل ارتباط

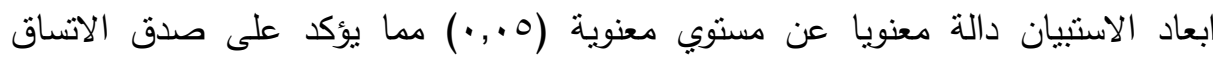

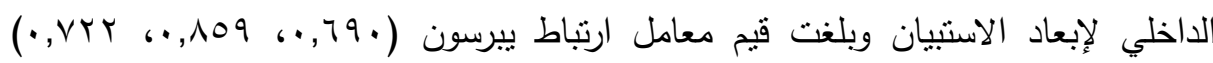

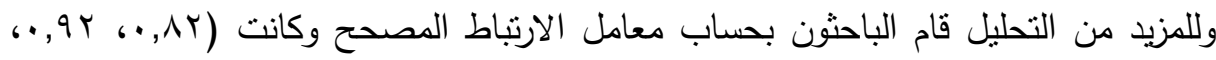

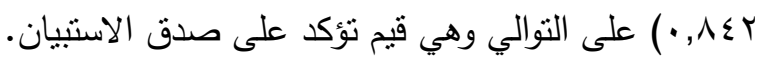

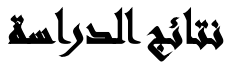

جدول رقم(ץ): توزيع عينة الدراسة لإستجابات عينة الدراسة على التساؤل هل يمكن ان يؤثز

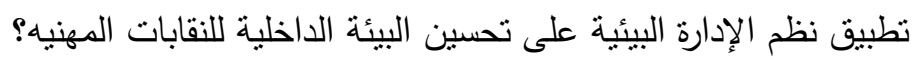

\begin{tabular}{|c|c|c|}
\hline النسبة & العدد & الإجابة \\
\hline 97,9 & $r \leqslant 7$ & نعم \\
\hline 1,7 & $\varepsilon$ & لا أعلد \\
\hline 1,7 & $\varepsilon$ & $\bar{y}$ \\
\hline$\% 1 \ldots$ & YOS & الإجمالي \\
\hline
\end{tabular}

من الجدول السابق لوصف عينة الدراسة تبعا لإستجابات عينة الدراسة على التساؤل هل يمكن ان يوثر تطبيق نظم الإدارة البيئية على تحسين البيئة الداخلية للنقابات المهنية كان عدد

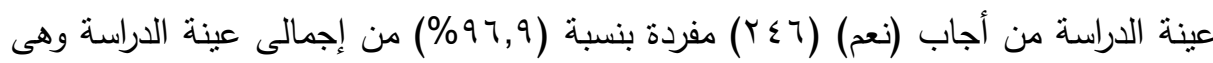

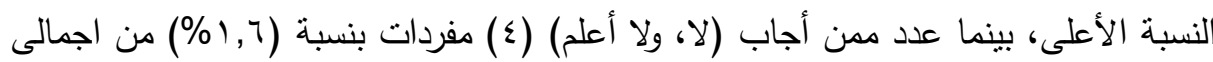




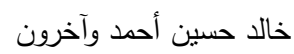

الفرض الأول: توجد علاقة ذو دلالة إحصائية بين تطبيق نظم الإدارة البيئية وتحسين أداء

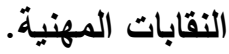
جدول(؟): اختبار بيرسون لتوضيح العلاقة الارتباطية بين نطبيق نظم الإدارة البيئية وتحسين أداء النقابات المهنية

\begin{tabular}{|c|c|c|}
\hline مستوى المعنويةة & معامل الارتباط (R) & تص \\
\hline$\cdot, \cdots 1$ & $\cdot, T \wedge Y$ & هنية \\
\hline
\end{tabular}

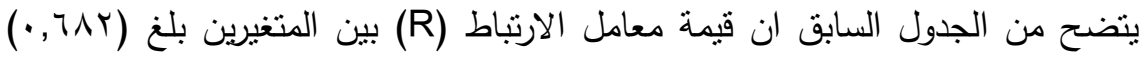

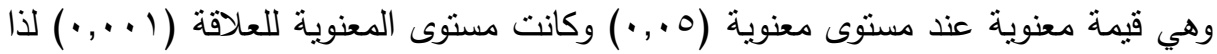
توجد علاقة ذو دلالة إحصائية بين تطبيق نظم الإدارة البيئية وتحسين أداء النقابات المهنية. لذا تتبت صحة الفرض الاول: توجد علاقة ذات دلالة إحصائية بين نطبيق نظم الإدارة البيئية

$$
\text { وتحسين أداء النقابات المهنية. }
$$

جدول رقم(؛) ): توزيع عينة الدراسة لإستجابات عينة الدراسة عن الأسباب التى تجعل النقابات المهنية لا تقوم بتطبيق نظم الادارة البيئية

\begin{tabular}{|c|c|c|}
\hline النسببة & العدد & الإجابة \\
\hline 77,1 & 171 & عدم اهتمام آدارات النقابات المهنية بتضمين نظم الأدارة البيئية فى \\
\hline$T Y, Y$ & 101 & غباب الوعى البيائى لدى ادارات النقابات المهنية \\
\hline $7 \cdot, 7$ & $10 \leqslant$ & غياب الضغوط لنطبيق نظم الإدارة البيئية \\
\hline 00,1 & $1 \leq$. & عدم توافر الموارد المالية اللازمة للدى النقابات المهنية لتطبيق نظم \\
\hline$r \cdot, V$ & $\vee \wedge$ & كل ما سبق \\
\hline
\end{tabular}

تم اختبار اكثر من أجابة من الجدول السابق لوصف عينة الدراسة لإستجابات عينة

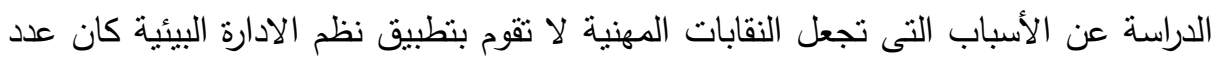

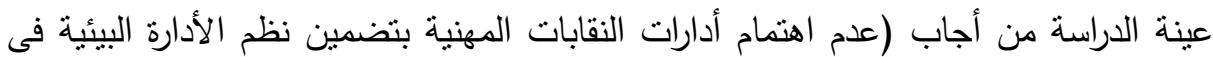

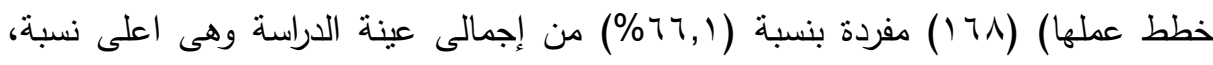

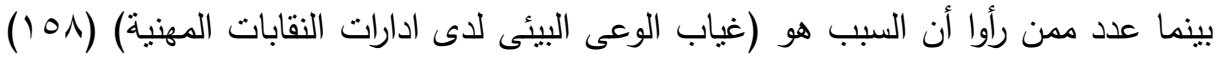

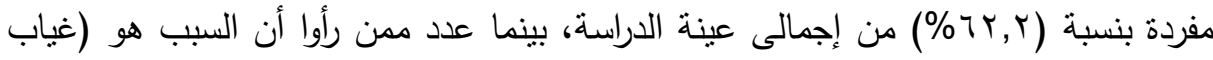

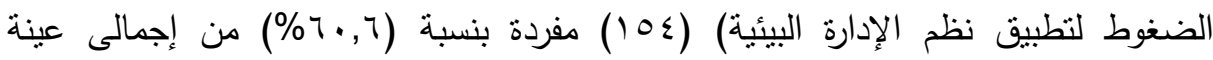

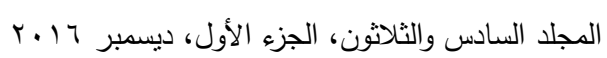


الدراسة، كان عدد عينة الدراسة من أجاب (عدم توافر الموارد المالية اللازمة لاى النقابات

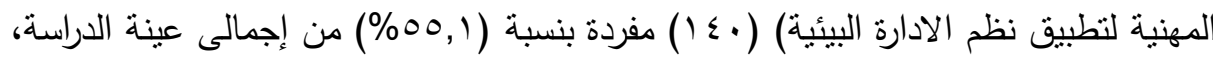

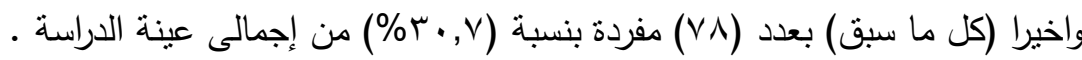

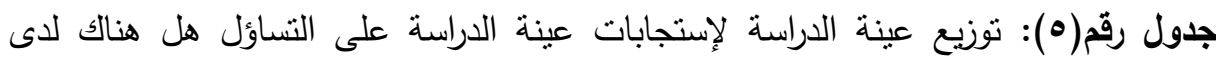

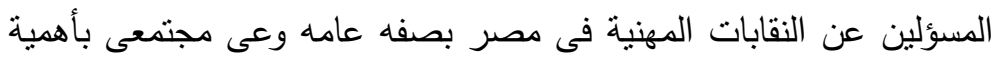

الحفاظ على البيئة؟ - مانه

\begin{tabular}{|c|c|c|}
\hline النسبة & العدد & الإجابة \\
\hline$\vee 9,7$ & $r \cdot r$ & $\bar{y}$ \\
\hline$r \cdot, 0$ & Or & نعم \\
\hline$\% 1 .$. & TOS & الإجمال \\
\hline
\end{tabular}

من الجدول السابق لوصف عينة الدراسة تبعا لإستجابات عينة الدراسة على التساؤل هل

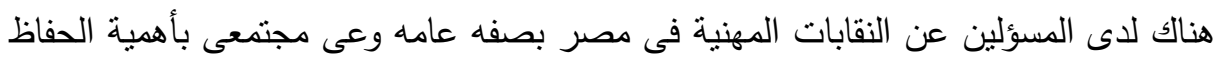

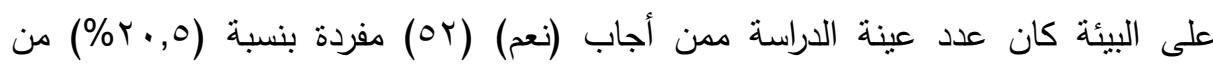

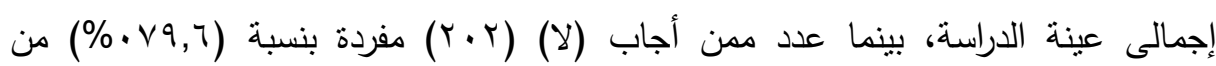
إجمالى عينة الدراسة وهى الأعلى.

الفرض الثاني: توجد علاقة ذات دلالة احصائية بين الوعى المجتمعى البيئي لاى إدارات النقابات المهنية ومعوقات تطبيق نظم الإدارة البيئية. جدول(†): اختبار بيرسون لتوضيح العلاقة الارتباطية بين الوعى المجتمعى البيئي لدى إدارات النقابات المهنية ومعوقات نطبيق نظم الإدارة البيئية

\begin{tabular}{|c|c|c|}
\hline مستوى المعنوية & معامل الارتباط ( R ) & الوعى المجتمعى البيئي لادى ادارات النقابات \\
\hline$\overline{\cdot, \ldots}$ & 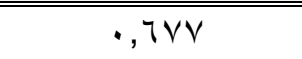 & ومعوقات تطبيق نُظٍ الإدارة البيئية \\
\hline
\end{tabular}

يتضح من نتائج تحليل الانحدار الخطى البسيط فى الجدول السابق ان قيمة معامل

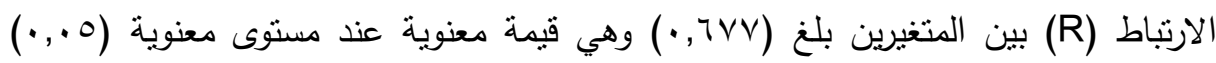

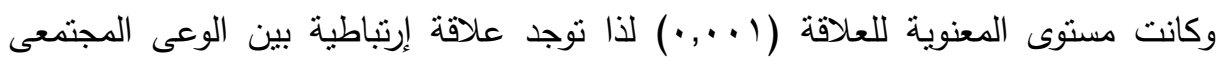
البيئى ومعوقات نطبيق نظم الادارة البيئية. 


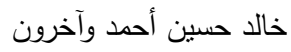

تثبت صحة الفرض الثانى: توجد علاقة ذات دلالة إحصائية بين الوعى المجتمعى البيئى ومعوقات تطبيق نظم الإدارة البيئية.

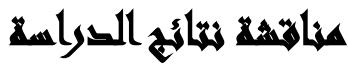

يتضح من نتائج اختبار بيرسون لتوضيح العلاقة الارتباطية ان قيمة معامل الارتباط

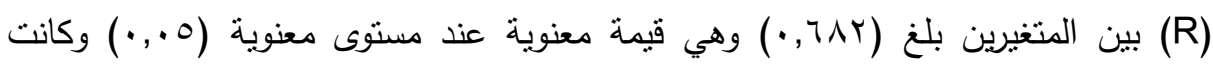
مستوى المعنوية للعلاقة ( ( .,. •) لذا توجد علاقة ذات دلالة إحصائية بين تطبيق نظم الإدارة البيئية وتحسين أداء النقابات المهنية. لذا تثبث صحة الفرض الاول: توجد علاقة ذات دلالة إحصائية بين نطبيق نظم الإدارة البيئية وتحسين أداء النقابات المهنية.

حيث ان تطبيق الادارة البيئية يؤدى الى التطوير والتحسين المستمر للاداء وتقديم

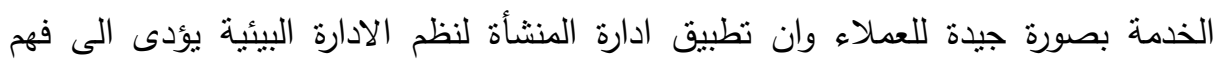

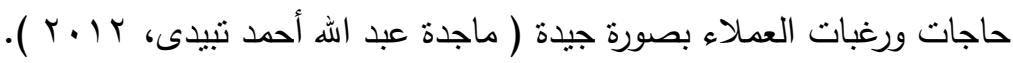

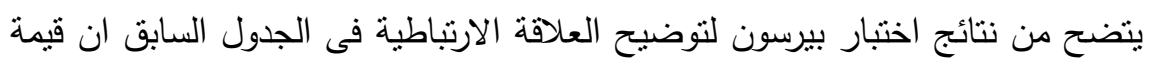

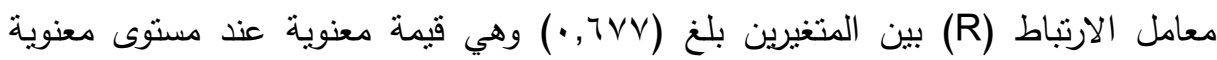

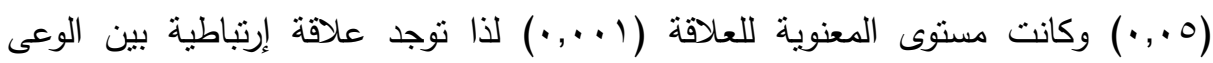
المجتمعى البيئى ومعوقات تطبيق نظم الادارة البيئية .

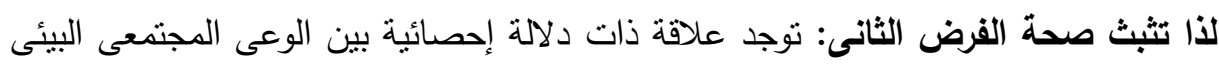

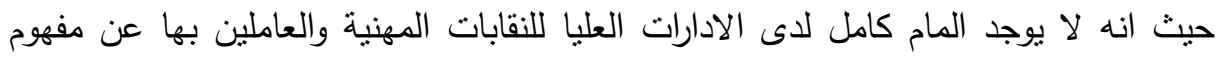
الادارة البيئية وأهدافها وسياستها ومنطلبات تحقيقها، بجانب ضعف الانه الاحساس لدى الادارة

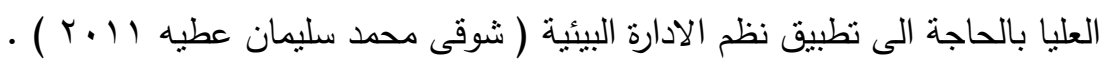

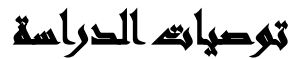

ا ـ تضمين الإدارة البيئية ضمن خطة عمل النقابات المهنية. r. تصميم نظام للأداء البيئي وذلك بوضع سياسة بئ بئئية والتأكيد على الالتزام بها.

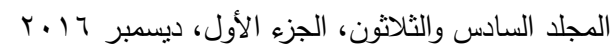


r. رفع وزيادة الوعى البيئى لدى المسئولين والعاملين بالنقابات المهنية عن طريق توفير برامج بيئية تنقيفية بأخطار أضرار تلوث البيئة، وأهمية وكيفية الحفاظ على البيئة.

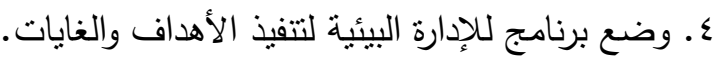

\section{xim}

ا ـ ـ توفير دعم من مؤسسات الدولة ومؤسسات المجتمع المدني.

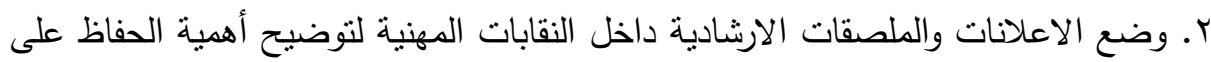

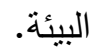

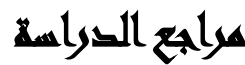

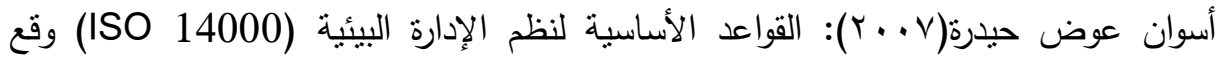
ومعوقات تطبيقها في المنظمات اليمنية، رسالة ماجستير ، كلية العلوم الادارية،

$$
\text { جامعة عدن }
$$

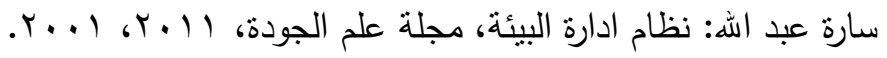

سيد الهوا ري(ع ـ . †): دليل الباحثين في إعداد البحوث العلمية، مكتبة عين شمس، القاهرة،

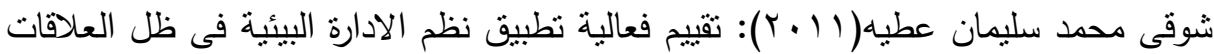

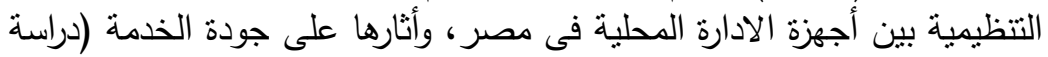

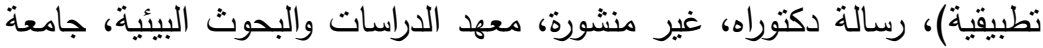

صلاح محمد الحجار ، داليا عبد الحميد صقر ، نظام الإدارة البيئية والتكنولوجية، دار الكتاب

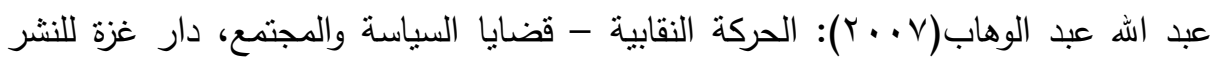
والتوزيع

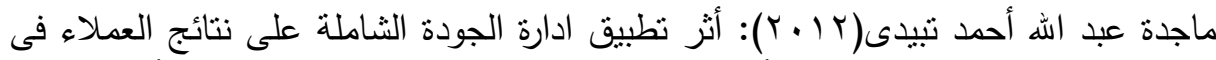

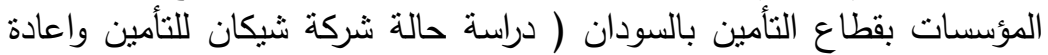

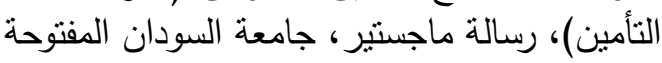
مجلة البحوث القانونية والاقتصادية: جامعة المنصورة، العدد 0 1، ابريل، 990 1.

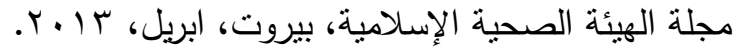


خالد حسين أحمد وآخرون

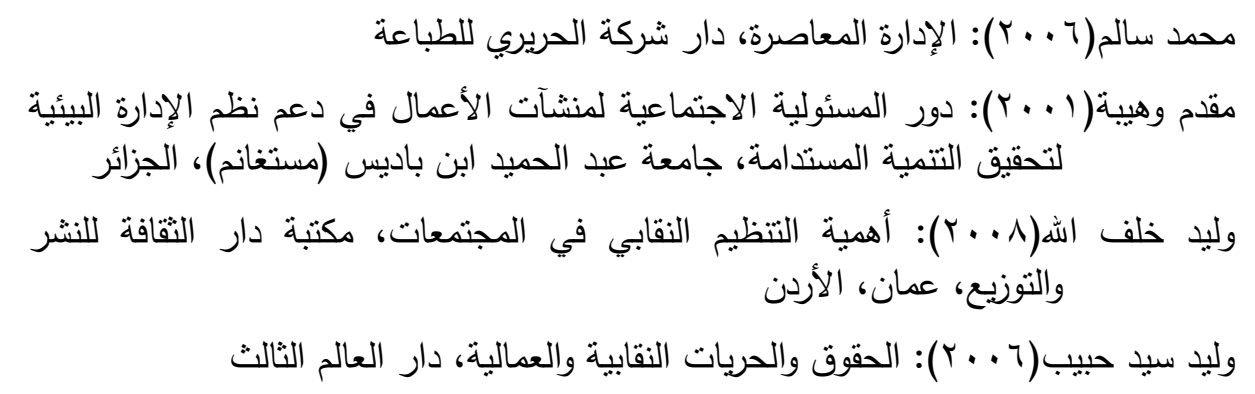

Apter, David, Introduction in standards performance organization and how to measure it ,Journal of Political analysis, (2006), vol (33), No, pp 141-172.

Carter tony, the Importance of training before working, the graduate school university of Wisconsin, 2009.

\title{
A PROPOSED PART FOR THE IMPLEMENTATION OF ENVIRONMENTAL MANAGEMENT SYSTEMS IN PROFESSIONAL ASSOCIATION
}

\author{
Ahmed, Kh. H. ${ }^{(1)}$; Mahmoud, M. S..$^{(1)}$ and Abdou, E. A. ${ }^{(2)}$ \\ 1) Faculty of Commerce, Ain Shams University 2) The General Trade \\ Association.
}

\begin{abstract}
The researcher drives at through this current study to set a proposed framework for applying the environmental management systems in trade syndicates, exploring as well the role of these environmental management systems in improving performance of trade syndicates.

Launching from the sense of social responsibility laid upon shoulders of trade syndicates and their members for being members in those syndicates, the study derives its significance from presenting an understanding of the active and effective role the environmental

$$
\text { المجلد السادس والثثلثون، الجزء الأول، ديسمبر } 17 \text {. }
$$


management systems play by standing on the essence and impact of those systems on trade syndicates' performance, attempting as well to identify the aspects of this influence on trade syndicates and the societal role for being considered as one of the civil societies' institutions in Egypt.

The study hypotheses show that there is a significant correlation between applying the environmental management systems and improvement of professional syndicates' performance. There is also a significant correlation between environmental societal awareness in professional syndicates' administrations and the obstacles of applying the environmental management systems. The study is concerned with defining shortness aspects in applying environmental management systems using the inductive method to prepare the theoretical framework to review previous studies, Arabic and foreign references. The study uses the descriptive (qualitative)-analytical method through a questionnaire form. The study is applied on a sample of (254) individuals from heads and members of syndicates' councils and occupational syndicates administrations, being applied on (24) syndicates in Egypt.

The study proves validity of hypotheses that: there is a relationship between administering environmental management systems and improvement of trade syndicates' performance and that there is a relationship between environmental societal awareness of trade syndicates and obstacles of applying the environmental management systems. Study recommendations recommend the necessity for including environmental management within the work plan of trade syndicates, recommending also with raising and increasing awareness of officials and employees of trade syndicates through exposing environmental cultural programs to enlighten them with risks of environment' pollution in addition to the importance and means of conserving the environment. 